\title{
XXIII.
}

Aus der chirurgischen Klinik zu Strassburg i. E.

\section{Ein Fall von Einklemmungsshok nebst Darmparalyse bei Hernia inguin. ext. incarcerata. ${ }^{1}$ ) \\ Von}

Dr. Hermann Trier.

Krankengeschichte.

Anamnese. Norbart Schnorr, 67 Jahre alt, Bauer aus Gamshausen. Seit dem 14. Juni 1889 hatte Patient keinen Stuhlgang mehr und konnte keine Winde mehr lassen. Am 16. Juni begann der bis dahin unempfindliche rechtsseitige Leistenbruch zu schmerzen. Am 17. Juni gegen Abend trat Erbrechen ein, welches fortdauerte. Das Erbrochene ist dunkelbraun, riecht säuerlich und ist ohne Fäcalbeimengung.

Patient giebt an, dass er den Bruch schon sehr lange besitze, dass er ihn aber bis zum letzten Jahre habe reponiren können. Der wegen der eingetretenen Schmerzen consultirte Arzt habe sehr lange und äusserst intensive Taxisversuche gemacht, jedoch ohne Erfolg. Deshalb stellt sich Patient am 18. Juni in der chirurgischen Klinik vor. Er klagt über heftige Schmerzen in der Bruchgeschwulst und im Leib.

Status praesens am 18. Juni. Alter magerer Mann, gelbe Hautfarbe. Das Gesicht schmerzhaft verzogen, mit dem Ausdrucke der Angst. Athmung frequent, jedoch keine objective Dyspnoe. Puls klein, sehr frequent. Leib etwas druckempfindlich, wenig aufgetrieben; Percussion ergiebt nirgends Dämpfung.

Herz und Lunge normal. Die rechte Scrotalhälfte ist zu Strausseneigrösse ausgedehnt. Auf der Kuppe des Tumors befinden sich Lymphome, von Contusionen und Taxisversuchen herrührend. Der Scrotaltumor lässt sich nach dem Leistenkanal und der Bauchhöhle hin verfolgen. Samenstrang nur undeutlich hinter der vorliegenden Bruchgeschwulst zu fühlen.

Die Hant iiber der Hernie fühlt sich fester an, als normal; keine entzuindliche Infiltration in derselben. Bruchgeschwulst ziemlich weich. Der Inhalt scheint geblähter Darm zu sein. Bruchwasser der Schätzung nach reichlich. Kein tympanitischer Schall durch Percussion nachzuweisen. Transparenz nicht vorhanden, Testikel undeutlich fuhlbar. - Bruchgeschwulst lässt sich nicht reponiren und ist äusserst schmerzhaft.

1) Auszug aus der gleichnamigen Dissertation. Strassburg i. E. Heitz und Mündel. 1890. 
Diagnose. Incarcerirte rechtsseitige Inguino-Scrotalhernie.

Therapie 18. Juni. Nachdem die ganze Umgebung der Genitalien rasirt und mit Seife gereinigt, wird Patient katheterisirt und erhält ein Vollbad. Taxisversuche ohne Erfolge.

Es wird Eis auf die Bruchgeschwulst gelegt. Anch nach Abkïhlung der Banchdecke bleiben Taxisversuche ohne Erfolg. Dieselben werden allerdings sehr wenig energisch gemacht, weil sie sehr schmerzhaft sind.

Nachmittags wird Patient narkotisirt mittelst Morphium und Chloroform. Da die Taxisversushe in der Narkose ebenfalls ohne Erfolg bleiben, wird zur Herniotomie geschritten.

Schnitt über die Höhe und ganze Länge der Bruchgeschwulst. Nach Eröffnung des Bruchsackes fliesst reichliches, geruchloses und nur wenig getrübtes Bruchwasser ab. Spaltung des Bruchsackes nach unten bis zur Kuppe des Scrotums, nach oben bis zum inneren Leistenring. Débridement der einschnürenden Stelle, stumpfe Erweiterung.

In dem eröffneten Bruchsack liegt der Testikel, sowie eine ziemlich derbe, mit Fibrinauflagerungen versehene röthlich blaue, noch spiegelnde Dünndarmschlinge; in derselben kein Koth.

Es handelt sich also um einen angeborenen Bruchsack. Der Darm wird aus der Bauchhöhle herausgezogen und die Bruchstelle inspicirt: verdächtige Schnürfurche. Reposition des Darms bis auf den Theil, welcher die verdächtige Schnürfurche trägt.

Desinfection der Darmschlinge mit 1 pro mille Sublimatlösung. Die Wunde wird offen gelassen, durch das Mesenterium ein undurchlöchertes Drainrohr geführt. Bedeckung der Darmschlinge mit Silk, der übrigen Wunde mit Jodoformgaze und Mousseline.

Temperatur Abends 37,80 C. Schmerzen bestehen noch. Erbrechen geringer. Schlechter Puls, 120 Schläge in der Minute.

19. Juni Morgens Temperatur $37,5^{\circ} \mathrm{C}$. Lebhaftes Brechen. Puls kleiu, frequent (125 Schläge). Magenausspülung, worauf Patient sich wohler fühlt. Opium. Abends Status idem. Temperatur 37,80 C. Fortgesetztes Brechen.

20. Juni Morgens Temperatur $37^{\circ} \mathrm{C}$. Entfernung der Drainröhre aus dem Mesenterium. Verband wie oben.

Nachmittags massenhaftes Erbrechen. Kalte Extremitäten. Puls immer schlechter. Collaps zunehmend. Abends Temperatur $37,4^{\circ} \mathrm{C}$.

21. Juni Morgens 1/1/2 Uhr Tod im Collaps. Keine deutlichen Sym. ptome von Peritonitis.

Section (von Herrn Prof. v. Recklinghausen ausgeführt) ergab Folgendes.

Etwas Struma. Varicen längs der V. saphena. Spannung der Bauchdecken. Grüne Färbung. Rechts liegt der mit grüner Gaze bedeckte Darm in grosser Ausdehnung zu Tage.

Mesenterium dieser Schlinge $7 \mathrm{Cm}$. lang; Schlinge am Darm gemessen $45 \mathrm{Cm}$. lang, mit Gasen stark gefutllt, Serosa spiegelnd, nur am prominentesten Theil matt graugrün. Auch am Mesenterium grünliche Färbung. Nur der Schenkel der Schlinge, der Bauchpforte der Operationswunde anflagernd, hat rothe Farbe.

Operationswunde bis zur Spitze des Scrotums $20 \mathrm{Cm}$. lang. Im unteren 
Fall von Einklemmungsshok nebst Darmparalyse bei H. inguin. ext. incarc. 563

Theil liegt Hoden und Samenstrang frei zu Tage. Die Oberfläche desselben geröthet. Ueber ihm liegt der Bruchsack, zum Theil stark geröthet und dickwandig, tibergehend in die Tunica propria, welche ebenfalls stark verdickt ist.

In der Bauchhöhle röthliche Flüssigkeit, nur wenig trüb. Wenig rothe kleine Blutgerinnsel. Gefässschlingen zum Theil stark injicirt; oberflächliche Injectionen vorhanden; kleine Extravasate an den venösen Gefässen bis zum Uebertritt auf das Mesenterium.

Ansammlung blutiger Flüssigkeit in den tiefsten Theilen des kleinen Beckens; nicht ganz $100 \mathrm{Ccm}$.

Beginnende Verknöcherung der Rippenknorpel, rothe Flüssigkeit in der Pleura. Blutige Flüssigkeit im Herzbeutel. Herz schlaff, braun, blutige Imbibition, etwas braune Atrophie des Herzfleisches. Beginnende Fäulniss. Cruormassen im Herzen.

Starke Blähung der Lungen. Rechte Lunge mehrfach adhärent. In den Bronchien bräunliche Flüssigkeit, offenbar Mageninhalt. Dunkelrothe Herde in den Unterlappen beider Lungen. Auch graue Herde dabei. Auf dem Schnitt sind diese bronchopneumonischen Herde trocken.

Schlaffe blasse Milz, runzelige Kapsel, geringe Vergrösserung. Stark geblähter Magen. Duodenum stark gebläht. Das Omentum majus bildet einen Strang von $31 \mathrm{Cm}$., Narben daran. Unteres Ende desselben hinter der Bauchwand an der Bruchpforte.

Innerhalb der Bauchhöhle, hinter der Bruchpforte nach links liegt eine Darmschlinge, stark dunkelblau geröthet wie die äussere. Sie gehört dem unteren Theil des Ileum und zwar dem rückführenden Schenkel der vorliegenden Darmschlinge an. Ihr folgt normaler $20 \mathrm{Cm}$. langer Darm bis zur Klappe. Bruchpforte für zwei Finger durchgängig. Serosa etwas fleckig-hämorrhagisch geröthet, aber keine der Gangrän verdächtige Stelle. Serosa nur etwas verdickt. Douglas'scher Raum ohne Veränderung.

Linke Niere etwas klein, schlaff, keine circumscripten Atrophien. Ebenso rechte Niere. Lymphdrüsen im rechten Oberschenkel stark vergrössert, weiss.

Im Duodenum braune dünne Flüssigkeit. Im Magen kothig gefärbte und riechende Flüssigkeit, darin kleine weisse Körner. Oesophagus weit, stark aufgetrieben, enthält denselben kothigen Inhalt. Gallenblase sehr stark gefüllt, Ductus choledochus frei. Lebergewebe blass, schaumiges Blut, Gallengänge injicirt. Harnblase stark gefüllt, Urin etwas trtib.

Die Darmschlinge lässt sich sehr leicht zurückziehen. Keine scharfe Abgrenzung zwischen dem innen und aussen gelegenen Theile. Die eingeklemmte Darmschlinge wird zuerst entleert, sodann mit Wasser gefiullt. An dem rückführenden Schenkel der aussen gelegenen Schlinge bildet sich eine ganz kleine, etwas roth gefärbte Stelle von circa $5 \mathrm{Mm}$. Durchmesser, an der ein kleines Divertikel sich vorbuchtet. An der eingeklemmten Schlinge zeigt sich nichts Besonderes, ausser einem circulär verlaufenden, flachen Einriss der Schleimhaut, $2 \mathrm{Cm}$. lang, mit rothen Rändern. Ebenso befindet sich in der Serosa des oberen Theils des zuführenden Schenkels der vorliegenden Schlinge ein oberflächlicher Einriss von circulärem Verlauf, $4 \mathrm{Cm}$. lang, $2 \mathrm{Cm}$. breit. Schleimhaut hämor- 
rhagisch geröthet an einer Stelle, welche $10 \mathrm{Cm}$. von dem inneren Einriss der Schleimhaut entfernt ist.

Auf der Wandung der Aorta abdominalis sind Blutgerinnsel, deutlich verklebt, blasse Färbung. Im Dickdarm weiche Fäcalmassen.

Unser Fall bietet zwei Erscheinungen dar, welche der Aufklärung bedürfen. Zunächst wird durch das Sectionsergebniss die eigentliche Todesursache nicht aufgedeckt; sodann scheint den heftigen Ileussymptomen die pathologisch-anatomische Grundlage zu mangeln.

Betrachten wir zunächst den ersten Punkt, nämlich die Frage nach der eigentlichen Todesursache.

Peritonitis, Gangrän, tödtlicher Blutverlust waren nicht vorhanden. Die Organe wurden bei der Section durchaus normal befunden, mit Ausnahme der Lungen. Diese enthielten in den Unterlappen geringe bronchopneumonische Herde. Da sich Mageninhalt in der Trachea vorfand, so handelte es sich hier wobl um eine Fremdkörperpneumonie, die indess von solch geringer Ausdebnung und so kurzer Dauer war (3 Tage), dass ihr die Schuld am Tode nicht beigemessen werden kann.

Todesfälle bei Brucheinklemmung mit negativem Sectionsergebniss werden vielfach in der Literatur erwähnt. Schon Richter (1788) kennt diesen Ausgang der Incarceration, und Pitha (1846) misst die Schuld an dem oft plötzlichen Tode der Bruchkranken einer Paralyse des Bauchnervensystems und des Bruchstiuckes bei.

Diese Todesfälle sind sicherlich in Analogie zu bringen mit denen, welche nach heftigen Contusionen des Unterleibs, nach Bauchwunden ohne Verletzung der Eingeweide, selbst nach einfachen Quetschungen eintreten. Nach dem Vorgange der Engländer bezeichnet man derartige unerklärliche Zufälle mit dem Namen "Shok".

Die Grundlage für alle Erklärungen des "Shoks" bildet der Goltzsche Klopfversuch. Die Herzlähmung wurde als die schuldige Ursache erkannt. Nach H. Fischer, C. Hueter und Billroth ist der Shok aufufassen als ein paralysirender Einfluss bei einer plötzlichen und heftigen Nervenverletzung auf die Herzthätigkeit. Nach Bill roth ist reiner Shok theoretisch nicht von Schmerzempfindung begleitet, während heftiger Schmerz einen grossen, wenn auch indirecten Einfluss auf das Eintreten von Collaps hat. Ueberwältigende physische und psychische Eindrücke rufen Collaps hervor, während der Shok von der Einwirkung der Psyche unabhängig ist. Collaps ist Ausdruck für acut eintretende Depressionszustände des Centralnervensystems. 
Fall von Einklemmungsshok nebst Darmparalyse bei $H$. inguin. ext. incarc. $\quad 565$

Billroth stellt hiermit gewissermaassen einen principiellen Unterschied auf zwischen Shok und Collaps; andere Autoren scheiden beide Begriffe nicht so streng. Nach Leichtenstern beruht anch der Collaps auf einer reflectorischen Herzlähmung von den Darmnerven aus.

Die Billroth'sche Definition des reinen Shoks als maassgebend angenommen, möchte ich glauben, dass der eigentliche Collaps sich nur in quantitativer Beziehung ron ihm unterscheidet. Der Shok ist ein plötzlicher Collaps, er wirkt plötzlich und sehr acut. Ist der Organismus kräftig genug, die Folgen des Shokanpralls zu überwinden, und hört die Wirkung der den Shok verursachenden Momente nicht auf, dann treten leicht die oben erwähnten überwältigenden physischen und psychischen Eindrïcke in ihre Rechte und der Collaps stellt sich ein. Shok und Collaps unterscheiden sich durch die Wucht ihres Angriffs auf ein mehr oder weniger widerstandsfähiges Nervensystem, und demnach ist ihre Wirkung mehr oder weniger intensiv. Shok kann augenblicklich zum Tode führen, Collaps thut dies mehr allmählich, falls die ihn bedingenden Ursachen fortdauern. Das Ziel, auf welches Shok und Collaps hinsteuern, ist die Herzparalyse.

Bei der acuten Einklemmung kommt ein reiner Shok im Billroth'schen Sinne wohl nur äusserst selten vor. Grosse Schmerzhaftigkeit der Bruchgeschwulst ist ja stets eine Begleitserscheinung der Incarceration, und die Facies hippocratica spricht mehr als alles Andere für eine Betheiligung der Psyche. Immerhin erwähnt Dieffenbach 2 Fälle, welche sich dadurch auszeichnen, dass die beiden Patienten sofort nach der Einklemmung in "Sopor wie ein Apoplektischer oder schwer am Kopfe Verwundeter" fallen. Auch Verlangsamung des Pulses wurde bei ihnen constatirt.

Die nervösen Erscheinungen bei der acuten Einklemmung ähneln demnach bald dem Shok, bald zeigen sie einen mehr allmählichen Verfall der Kräfte. Der Tod tritt oft schon wenige Stunden nach der Incarceration auf, während er in anderen Fällen, wie z. B. in unserem, erst nach Tagen erfolgt. Leichtenstern hat daher die shokähnlichen Collapserscheinungen mit dem Gesammtnamen „Einklemmungsshok" bezeichnet.

Um dem Verständniss der genaueren Vorgänge beim Einklemmungsshok näher zu treten, müssen wir vom Goltz'schen Klopfversuch ausgehen. Bei demselben wird durch Schlagen des Darms das Herz vorübergehend zum Stillstand gebracht. Aus den Versuchen Bernstein's und vieler Anderer geht hervor, dass durch Reizung der nervösen Elemente des Unterleibs das Vaguscentrum erregt und dadurch die Herzthätigkeit gehemmt wird. Verlangsamung des Pulses 
ist damit verbunden, wie die oben genannten Dieffenbach'schen Fälle zeigen. Die Frage, welche Nerven im Einzelnen bei der Incarceration in Reizzustand versetzt werden, kann vor der Hand nicht beantwortet werden.

Der Verlangsamung steht in der weitaus grössten Zahl der Fälle von Einklemmungsshok eine Beschleunigung der Pulsfrequenz gegenüber. Die theoretische Erklärung dieser Erscheinung folgt ebenfalls aus dem Goltz'schen Klopfversuch. Bei diesem nämlich beobachtet man, dass sämmtliche Blutbahnen des Darmtractus sich stark füllen, während die Gefässe der Haut, der Extremitäten, kurz des ubbrigen Körpers mehr oder weniger blutleer werden. Goltz erklärt dies durch eine Lähmung des Tonus der Darmgefässe, welche hervorgerufen werden kann durch Reizung des Darms oder der Eingeweidenerven selbst.

Mit Hülfe dieser Erscheinung kann der Einklemmungsshok (auch für unseren Fall) verständlich gemacht werden. Durch die Einklemmungsinsulte, ferner durch die intensiven, erfolglosen Taxisversuche wurde der vasomotorische Darmnerv, der Splanchnicus, gelähmt und dadurch eine Erweiterung der Darmgefässe bewirkt. Die sehr zahlreichen Gefässe des Darms füllten sich stark mit Blut, oberflächliche Injectionen und kleine Extravasate an den Venen bịldeten sich, wie es bei starker Hyperämie im peritonealen Ueberzuge häufig der Fall ist. Die kalten Extremitäten, die kühle, blasse Haut, der kleine Puls bezeugten, dass die tubrigen Körpergebiete blutarm wurden. An der Anämie des Körpers nahmen auch das Gehirn und das Mark Theil : der Vagustonus wurde herabgesetzt und die Pulsfrequenz stieg bis auf 125 Schläge in der Minute.

Die Temperatur beim Shok ist in der Regel erniedrigt, einmal wegen der geringen in Circulation befindlichen Blutmenge, sodann vielleicht, weil auch hier nervöse Beeinflussung sich geltend macht. In unserem Falle schwankt die Temperatur zwischen 37,8 und 37,0 . Als Collapstemperaturen sind solche Zahlen etwas hoch; doch erklärt sich diese Erscheinung leicht, wenn man die bestehende Bronchopneumonie als Ursache der erhöhten Temperatur betrachtet.

$\mathrm{Zu}$ diesem Bilde des Einklemmungsshok gesellen sich noch einzelne Züge, welche die Prognose um so mehr zu einer ungünstigen gestalten, je höher das Alter des Patienten ist und je mehr derselbe durch die Unmöglichkeit einer ausreichenden Ernährung geschwächt ist. Es sind zu nennen: die nervöse Ueberreizung, die dadurch entstehende gewaltige Erschöpfung der Kräfte, die psychische Depression des Patienten, welcher sich in höchster Lebensgefahr weiss, und das 
Fall von Finklemmungsshok nebst Darmparalyse bei H. inguin. ext. incarc. 567

Erbrechen. Von mancher Seite (Reichel, Bellon) wird gerade diesen Symptomen die Hauptscbuld am letalen Ausgange beigemessen. Durch die Einklemmungsinsulte soll mit Hülfe des N. vagus dexter und der Nn. phrenici das Gehirn in einen reflectorischen Depressionszustand versetzt werden, welcher alsdann den Tod verursacht. Wenn auch zugegeben werden kann, dass beim Einklemmungsshok eine solche directe Beeinflussung des Gehirns möglich scheint, so sprechen doch sowohl die klinischen Erscheinungen, als auch der Sectionsbefund entschieden für die Herzparalyse als eigentlicher Todesursache. -

Gehen wir nunmehr zu dem zweiten Punkt bei unserem Falle uber, welcher der Erläuterung bedarf.

Der Patient erkrankte an acuter Brucheinklemmung, welche, wie stets, von lebhaftem, bis zum Ileus gesteigertem Brechen begleitet war. Die Herniotomie wurde gemacht und die Einklemmung gehoben. Gleichwohl dauerte das Erbrechen, und zwar in gesteigertem Maasse fort bis zum Tode. Die Section ergab kein mechanisches Hinderniss in der Kothpassage, speciell die incarcerirt gewesene Schlinge war völlig durchgängig. Koprostasen waren nicht vorhanden, Gangrän bestand nicht.

Da ein mechanisches Hinderniss nicht vorhanden war, müssen wir ein functionelles annehmen. Als solches ist das Aufhören der Peristaltik zu betrachten.

Fälle, in denen eine derartige Erscheinung: zur Beobachtung kam, sind sebr selten. Bei zwei ähnlichen Fällen, welehe von $G$ ay und von Bond beschrieben wurden, waren die betreffenden Verfasser genöthigt, eine Darmparalyse als Ursache des bis zum Tode dauernden Ileus anzunehmen. Auch für unseren Fall müssen wir diese Diagnose beanspruchen. Um dieselbe näher begrtinden zu können, wollen wir uns nach den ätiologischen Momenten umsehen, welche eine Darmlähmung hervorzurufen vermögen.

Atonie und Paralyse des Darms entstehen am gewöhnlichsten bei langdauernder Obstipation, z. B. bei chronischem Darmkatarrh. Es bilden sich Koprostasen, welche einerseits die Stuhlentleerung mechanisch verhindern, andererseits durch Dehnung der Musculatur und Verdrängung des ernährenden Blutes die Elasticität der Darmwand vernichten können. Die Peristaltik wird abgeschwächt und kann schliesslich ganz aufgehoben werden. In gleicher Weise kann der Darm experimentell durch starke Ausdehnung mittelst Gasen gelähmt werden.

Darmlähmung wird ferner bei typhösen, tuberculösen und dysenterischen Erkrankungen des Darms beobachtet. Auch Peritonitis kann Darmschwäche und auch wohl Paralyse hervorrufen. 
Endlich bat man nach schweren Verletzungen des Unterleibs, z. B. nach Pferdehufschlag, die Symptome des Darmverschlusses gesehen, ohne bei der Autopsie ein mechanisches Hinderniss finden zu können.

Von allen erwähnten ätiologischen Momenten besitzt nur die Paralysirung des Darms durch Trauma des Unterleibs Aebnlichkeit mit der Brucheinklemmung. Der Schlag durch den Pferdehuf und die Einklemmungsinsulte, noch verstärkt durch die intensiven Taxisversuche, sind in ibrer lähmenden Wirkung auf den Darm in Parallele zu setzen. Wie ist diese lähmende Wirkung bei der Brucheinklemmung zu erklären?

Nach den Untersuchungen von Schwenninger and Kocher erlischt in einer durch einen Ring gezogenen und sodann stark aufgeblähten Darmschlinge die Peristaltik. Nach Entfernung des Ringes dauert die Lähmung noch eine Zeit lang fort und zwar um so länger, je längere Zeit hindurch der venöse Rückfluss oder gar der arterielle Zufluss gehemmt war. Die Wände des Darms werden durch den Druck des Inhalts mehr oder weniger blutleer gemacht und dadurch in ihrer Ernährung gestört; vor Allem aber wird die Musculatur durch die Dehnung für einige Zeit ihrer Elasticität beraubt.

Reichel theilt der Dehnung nur eine nebensächliche Rolle zu und bält die durch Compression der Gefässe im Bruchring bedingten Circulationsstörungen für die eigentliche Ursache der Darmlähmung.

Für unseren Fall sind beide Momente heranzuziehen. Die Schlinge zeigte eine blaurothe Farbe, Bruchwasser war reichlich vorhanden beides Zeichen von Circulationsstörungen. Ferner war die Schlinge mit Gasen gefüllt, also auch bis zu gewissem Grade gedelnt. Da übrigens nach Reichel mehrfach wiederholte forcirte Taxisversuche ebenfalls Circulationsstörungen bewirken können, so ist auch dieser Umstand bei unserem Falle zu berïcksichtigen.

Die Frage nach der eigentlichen Wirkungsweise der Circulationsstörungen lässt sich, glaube ich, mit Heranziehung eines weiteren Momentes in Folgendem beantworten.

Nach den Untersuchungen von Pflüger bewirkt Reizung des Splanchnicus einen fast augenblicklichen Stillstand der Peristaltik des Dünndarms, und zwar in der Diastole der Musculatur. Der Splanchnicus ist der vasomotorische Nerv des Darms; durch seine Reizung. wird die Blutzufuhr vermindert. Die Peristaltik wird also gehemmt, wenn die im Blut befindlichen Reize nicht mehr dem Darm zufliessen.

Nach meiner Meinung lässt sich danach die Paralyse in unserem Falle erklären. Die vorliegende Schlinge war ziemlich gross, so dass 
Fall von Einklemmungsshok nebst Darmparalyse bei H. inguin. ext. incarc. $\quad 569$

das Mesenterium eine hinreichende Menge der den Darm versorgenden Splanchnicusfasern enthielt. Die acute Einschnürung, welche eine tiefe Schnürfurche in die Darmwand grub, diente als intensive Reizung dieser Fasern und hatte demgemäss Darmparalyse zur Folge. Da diese Lähmung des Darms nach v. Basch in letzter Linie auf einer reflectorisch erzeugten Verminderung der Blutzufuhr beruht, so muss die durch den Bruchring bewirkte directe Compression der Arterien in demselben Sinne wirken, wie die Splanchnicusreizung. Auch der Erfolg der Darmdehnung, welche ja nicht bedeutend genug war, um an sich die Elasticität der Musculatur zu vernichten, ist auf Rechnung der Verdrängung des arteriellen Blutes zu setzen. Nicht die venöse Stauung lähmt den Darm - denn Venosität des Bluts erregt im Gegentheil die Peristaltik -, sondern das Fehlen des arteriellen Bluts und der in diesem befindlichen specifischen Reize. Wir haben demnach drei Momente, welche alle in derselben Richtung thätig sind, nämlich: Dehnung des Darms, Bruchringeompression und Splanchnicusreizung.

Die lange Dauer der Lähmung wird aus den Pflüger'schen Experimenten verständlich. Aus denselben folgt, dass nach Aufhören der Splanchnicusreizung die Darmbewegung nicht unmittelbar wieder beginnt, sondern dass eine beträchtliche Nachwirkung besteht. Diese macht sich um so länger geltend, je intensiver die Reizung war, und je schwächer die peristaltischen Bewegungen vor der Reizung waren.

Die Einklemmung, welche eine tiefe Schnürfurche in die Darmwand grub, besonders aber die Taxisversuche übten sicherlich eine ganz gewaltige Nervenreizung aus. Diese, selbst wenn sie nur kurze Zeit andauerte, genügte, um die Schlinge auf längere Zeit hinaus zu lähmen. $\mathrm{Da}$ in alten Brüchen die Peristaltik schon von vornherein eine abgeschwächte ist, musste die Wirkung der Splanchnicusreizung um so nachhaltiger sein. Daher bestand die Darmparalyse auch nach der Herniotomie fort.

Wir haben oben gesehen, dass der Einklemmungsshok durch Gehirnanämie und Herzparalyse infolge ron reflectorischer Lähmung der vasomotorischen Splanchnicusfasern hervorgerufen wird. Zur Erklärung der Darmlähmung aber nahmen wir eine Splanchnicusreizung an. Beide Erscheinungen lassen sich in in folgender Weise mit einander vereinigen. Die acute Einklemmung verursachte zunächst eine Reizung des Splanchnicus. Infolge der obne entsprechende Ruhepausen andauernden übermässigen Erregung des Nerven trat eine Erschöpfung desselben ein, welche die Lähmung des Darmgefässtonus mit ihren Folgen veranlasste. 
Die Erscheinung des Ileus bei Darmparalyse lässt sich durch van Swieten's Theorie einfach erklären. Diese besagt in Kürze, dass der Darminhalt nach dem Orte des geringsten Widerstandes hin ausweicht. Da das paralysirte Darmstick ein functionelles Hinderniss darbietet, wird der Koth unter Mitwirkung der Bauchpresse und des durch Gase erhöhten intraabdominellen Druckes nach dem Magen zu gedrängt.

Ausser der Darmparalyse sind noch zwei andere Ursachen für das Erbrechen vorhanden. Die Einschnürung an sich stellt durch Verlegung des Darmlumens ein mechanisches Hinderniss dar und verursacht Ileus. Derselbe wird durch die Herniotomie beseitigt und ist nicht Gegenstand unserer Besprechung. Sodann haben wir unter den Symptomen des Einklemmungsshok gleichfalls Erbrechen genannt, welches natürlich, wie der Shok, nach der Operation nicht schwand.

Dies Shokerbrechen hat mit der Affection des Darms an sich nichts zu thun. Es tritt auch dann auf, wenn gar kein Darmbruch vorliegt. Mehrfach ist Erbrechen, selbst Miserere bei reinen Netzbrüchen beobachtet worden. Ferner behandelten Kussmaul und Li i cke mehrere kleine Fetthernien in der Linea alba, welche neben intensiven Schmerzen heftiges Erbrechen verursachten. Dieses Brechen ist als eine Reflexerscheinung zu betrachten, ausgehend von einer Reizung der nervösen Elemente des Unterleibs. Gleichwie das Brechen infolge der Darmparalyse, ist das Shokerbrechen kein Product der Antiperistaltik, sondern ist allein auf die Bauchpresse und den erhöhten Druck in den Därmen zurtickzuführen. Reflectorisch werden die Bauchmuskeln zur Contraction gebracht in ganz ähnlicher Weise, wie auch andere Muskelgruppen. Muskelkrämpfe der verschiedensten Körperregionen bilden einen Theil jener mannigfaltigen nervösen Erscheinungen bei acuter Incarceration, wie sie Lapeyre zusammengestellt hat. -

Fassen wir die Erscheinungen zusammen, welche unser Fall bietet, so erhalten wir folgendes Resultat. Als Todesursache ist der Einklemmungsshok anzusehen, d. h. der Gesammteffect aller von der Reizung des Unterleibs reflectorisch hervorgerufenen abnormen Einwirkungen des Centralnervensystems, in erster Linie also die Paralyse des Herzens. Der Shok hatte im Gefolge Ileuserscheinungen. Unabhängig von Shok, weil durch rein locale Einwirkung bedingt, bestand reflectorische Darmparalyse. Letztere kennzeichnete sich durch unstillbaren Ileus, sowie durch den Sectionsbefund, welcher nachwies, dass trotz des Fehlens jeglichen mechanischen Hindernisses kein Darminhalt die völlig durchgängige Darmschlinge passirt hat. 
Fall von Eïnklemmungsshok nebst Darmparalyse bei H. inguin. ext. incarc. $\quad 571$

Hieraus geht hervor, dass die Diagnose der Darmparalyse mit Sicherheit nur post mortem gestellt werden kann, da nur die Section den Mangel eines mechanischen Hindernisses, sowie das Fehlen einer anderweitigen darmlähmenden Erkrankung mit Bestimmtheit nachweisen kann. Was die Diagnose des Einklemmungsshoks bei Lebzeiten des Kranken betrifft, so kommen folgende Erkrankungen in Betracht, welche ähnliche Symptome darbieten:

Altersanämie, sowie Fettembolie; sie sind bei unserem Patienten auszuschliessen.

Peritonitis und acute Septicämie; da beide Erkrankungen mit Fieber verbunden sind, so bildet dasselbe das wichtigste Unterscheidungsmerkmal zwischen ihnen und dem Einklemmungsshok. Die übrigen Symptome gleichen sich oft vollständig.

Auch mit Cholera ist der Einklemmungsshok schon verwechselt worden, besonders wenn bei der Einklemmung die sogenannte Choléra berniaire vorhanden war. Die Section wird in diesen Fällen das wahre Wesen der Krankheit enthüllen.

Abkühlung des Darms und des Peritoneums erzeugt experimentell Herzstillstand. Bei unserer heutigen Operationstechnik ist man bestrebt, diese Gefahr während der Operation dadurch zu vermeiden, dass man die freigelegten Eingeweide vor Erkältung schützt. Da lubrigens Kälte als Reiz auf die Nerven wirkt, so ist wobl kaum ein Grund vorhanden, theoretiseh die Erkältung des Bauchfells und seiner nervösen Apparate von dem gewöhnlichen Bauchshok zu trennen, wie es Nussbaum verlangt.

Was die Differentialdiagnose zwischen Einklemmungsshok und Tod durch Verblutung betrifft, so lässt sich dartiber Folgendes sagen. Wir haben den Einklemmungsshok in der Weise erklärt, dass eine durch Lähmung der vasomotorischen Splanchnicusfasern erzeugte Bauchhyperämie eine verderbenbringende Gehirnanämie hervorruft. Danach wirkt dieser Shok genau wie eine Verblutung; denn es ist gewiss gleichgtiltig, ob das Blut den Körper ganz verlässt, oder ob es in den zahlreichen Gefässen des Darmtractus zurïckgehalten und dem Kreislauf entzogen wird. Der Erfolg ist in beiden Fällen derselbe.

Zum Schlusse noch einige Worte über die Therapie.

Das souveräne Heilmittel bei Brucheinklemmung ist die Herniotomie. Zu warnen ist davor, die Zeit nutzlos mit der Darreichung von inneren Mitteln, wie Quecksilber, verstreichen zu lassen. Auch die Taxisversuche, zumal wenn sie lange und intensiv fortgesetzt werden, sind $\mathrm{zu}$ verdammen. Ich bin der Ueberzeugung, dass in 
unserem Falle gerade den forcirten und fruchtlosen Taxisversuchen eine nicht geringe Schuld an dem Einklemmungsshok und der Darmparalyse beizumessen ist.

War man genöthigt, eine verdächtige Darmschlinge ausserhalb der Bauchböhle zu lassen, so schütze man den Darm vor Erkältung. Ueberhaupt spielt die Wärmezufuhr bei Shokkranken eine sehr wichtige Rolle, sowohl während der Operation wie bei der Nachbehandlung.

Hört auch nach der Operation der Ileus nicht auf, so wird man, wie es in unserem Falle geschehen ist, seine Zuflucht zur Magenausspülung nehmen.

Ueber die Wirksamkeit dieser von $\mathrm{Kussmaul}$ eingeführten Heilmethode ist viel hin- und hergestritten worden. So lange es sich um die Anwendung der Magenpumpe bei noch bestehender Incarceration handelt, muss man der Ansicht von Bardeleben, Kümell u. A. beipflichten, welche dieselbe für nutzlos und geradezu gefährlich erklären. Sobald aber durch die Herniotomie das mechanische Hinderniss beseitigt ist, kann wohl kaum eine geeignetere Behandlungsweise des Ileus ersonnen werden, als die Ausspulung.

Nach den Untersuchungen von A. Cahn, Oser und Hasenclever besteht die Wirksamkeit der Magenpumpe darin, dass ausser dem Magen auch der Darm entleert wird. Ueber die weiteren näheren Vorgänge ist man noch nicht einig geworden. Die Hauptsache ist, dass die Magenausspülung eine überaus wohlthätige Wirkung auslibt, welche auch bei unserem Patienten zu bemerken war. Sie entlastet den Darm, entfernt den lästigen und den Kranken beängstigenden Meteorismus, und wirkt auch in psychischer Hinsicht dadurch günstig, dass die Fäces nicht mehr unmittelbar den Mund zu passiren brauchen. Dem Darm wird Zeit gegeben, sich in Ruhe zu erholen.

Nach Pflüger's Untersuchungen kehrt die Peristaltik des auf reflectorischem Wege gelähmten Darms stets wieder. Unter der Einwirkung der Magenausspülung wäre auch in unserem Falle die Darmlähmung sicherlich gewichen, wenn der Einklemmungsshok nicht so lange nachgewirkt hätte. Gegen diesen aber ist das Kussmaul'sche Mittel erfolglos. 
Fall von Einklemmungsshok nebst Darmparalyse bei H. inguin. ext. incarc. 573

\section{Literatur.}

1. Richter, Abhandlung von den Brüchen. 1788.

2. Pitha, Prager Viertejjahrschrift. 1846.

3. Nussbaum, Quetschungen und Zerreissungen am Unterleib. 1882.

4. Goltz, Centralblatt. 1863 u. 1864.

5. Billroth, Handbuch der allgem. und spec. Chirurgie. 1878. Bd. I.

6. Leichtenstern, Prager Vierteljahrschrift. 1873.

7. Derselbe in Ziemssen, Spec. Pathologie und Therapie. VII, 2.

8. Dieffenbach, Operative Chirurgie. 1848. Bd. II.

9. Bernstein, Centralblatt. 1863 u. 1864.

10. Reichel, Lehre von der Brucheinklemmung. 1886.

11. Bello n, Des sympt. de l'étranglement int. d. 1. rapports av. 1. siège d. 1 . lésion. 1878.

12. Eichhorst, Die troph. Bez. d. Nn. vagi z. Herzmuskel. 1879.

13. Gay, G. W., Bost. med. and surg. Journ. 1886.

14. Bond, Lancet 1882.

15. Pflüger, Ueber das Hemmungsnervensystem für die perist. Bewegungen der Därme. 1857.

16. Beck, Herniologisches. Langenbeck's Archiv. Bd. XXX.

17. van Braam-Houckgeest, Pflüger's Arch. f. Phys. Bd. IV.

18. NothnageI, Experimentelle Untersuchungen über Darmbewegungen. 1882.

19. Danzel, Herniologische Studien. 1863.

20. Lü cke, Centralblatt für Chirurgie. 1887.

21. Lap eyre, Les accid. nerv. que l'on observe dans l'étranglem. hern. 1880.

22. Nussbaum, Ueber Shok grosser Verletzungen und Operationen. Aerztliches Intelligenzblatt. 1877.

23. Bardeleben, Berliner klin. Wochenschrift 1885 .

24. Kü mell, Deutsche med. Wochenschrift 1886 .

25. Cahn, Berliner klin. Wochenschrift 1884 u. 1886.

26. Hasenclever, Ebenda. 1886.

27. Oser, Wiener med. Blätter. 1884. 